\title{
A gluten-free diet score to evaluate dietary compliance in patients with coeliac disease
}

\author{
Federico Biagi ${ }^{1}$, Alida Andrealli ${ }^{1}$, Paola Ilaria Bianchi ${ }^{1}$, Alessandra Marchese ${ }^{1}$, Catherine Klersy $^{2}$ \\ and Gino Roberto Corazza ${ }^{1}$ \\ ${ }^{1}$ Coeliac Centre/First Department of Internal Medicine, Fondazione IRCCS Policlinico San Matteo, University of Pavia, Piazzale \\ Golgi 19, 27100 Pavia, Italy \\ ${ }^{2}$ Biometry and Clinical Epidemiology, Fondazione IRCCS Policlinico San Matteo, University of Pavia, Piazzale Golgi 19, 27100 \\ Pavia, Italy
}

(Received 17 October 2008 - Revised 14 January 2009 - Accepted 9 February 2009 - First published online 31 March 2009)

A dietary interview performed by expert personnel is considered to be the most appropriate tool to check whether patients with coeliac disease follow a strict gluten-free diet. However, we currently have no straightforward and non-subjective method for performing such a dietary interview. We therefore developed a fast questionnaire based on four simple questions with a five-level score ( $0-$ IV). To verify whether our questionnaire is an efficient tool, we applied it to 168 coeliac patients (126 females and 42 males; mean age 42.4 (SD 12.9) years) on a gluten-free diet (median 82 , 25 th-75th percentile 50-108, range 15-389 months). The score we obtained was compared with the persistence of both villous atrophy and endomysial antibodies while on a gluten-free diet. A comparison with survival of the patients was also performed. Patients were interviewed over the phone by non-expert personnel. The questionnaire was completed in less than $1 \mathrm{~min}$. The lowest results were significantly more frequent among the patients with a persistence of both villous atrophy and positive endomysial antibodies. Death risk was also significantly correlated with the lowest score results. We conclude that our questionnaire is a reliable and simple method of verifying compliance with a gluten-free diet.

Coeliac disease: Gluten-free diet: Endomysial antibodies: Dietary compliance

Although a gluten-free diet (GFD) is extremely important for patients affected by coeliac disease ${ }^{(1,2)}$, it is a known fact that not all patients are able to follow it to a sufficient extent. According to some series, as many as $30-40 \%$ of coeliac patients do not follow a strict $\mathrm{GFD}^{(3)}$. While this may be understandable for some categories of patients, such as adolescents $^{(4-7)}$, it must be stressed that even the most careful adult patients may experience some initial confusion in dealing with a new type of diet, especially if they are not given sufficient information $^{(3)}$. It must also be pointed out that keeping to a strict GFD is difficult due not only to the lack of knowledge of the problem on the part of chefs and other individuals working in the catering sector ${ }^{(8)}$, but also to the fact that gluten may be present in packaged foods considered to be gluten-free ${ }^{(9,10)}$. On the basis of these observations, Collin et al. believe that an absolute and complete GFD is unrealistic ${ }^{(10)}$.

The problem of gluten contamination in apparently glutenfree foods has led to a series of studies aimed at understanding the minimum quantity of gluten that can cause damage to the intestinal mucosa ${ }^{(10-15)}$. An Italian study recently demonstrated that $50 \mathrm{mg} / \mathrm{d}$ for $90 \mathrm{~d}$ have a toxic effect on the intestinal mucosa of patients affected by coeliac disease ${ }^{(14)}$. Although this result can be considered highly alarming, if it is translated into terms of normally eaten quantities of food we can see that $50 \mathrm{mg}$ gluten are contained in food samples that cannot be eaten by mistake by a sensible and diet-conscious individual (Fig. 1, samples A, B, C and D). Vice versa, the amount of gluten contained in a crumb of bread small enough to be eaten by mistake (Fig. 1, sample E) is twenty times less than the $10 \mathrm{mg}$ gluten that did not show a toxic effect after $90 \mathrm{~d}$ consumption ${ }^{(14)}$. In addition, if we consider the limit of 20 parts per million (ppm) indicated by numerous coeliac patient associations ${ }^{(16-20)}$, i.e. $20 \mathrm{mg}$ gluten per $\mathrm{kg}$ product, then for histological damage to occur the coeliac patient would have to eat $2.5 \mathrm{~kg}$ of contaminated product per $\mathrm{d}$ for $90 \mathrm{~d}$. It is clearly impossible to eat this amount by mistake. Even if we take the limit of $100 \mathrm{ppm}$ considered safe by Collin et al. ${ }^{(10)}$, the coeliac patient would have to eat $500 \mathrm{~g}$ of contaminated product per $\mathrm{d}$ for $90 \mathrm{~d}$ to reach the toxic dose. It seems reasonable to think that such an involuntary mistake would be improbable. A directive has also recently been issued regulating the labelling of foodstuffs through the European Union $^{(16,21)}$. This directive obliges manufacturers to indicate the presence of gluten content, regardless of the amount. Similar directives also exist in Australia and the $\mathrm{USA}^{(17,19,22)}$. The application of this directive could mean another organisational difficulty for coeliac patients who may prefer to avoid eating safe packaged products, in that they contain gluten but the amount is below the 20 ppm threshold.

Abbreviations: EMA, endomysial antibodies; GFD, gluten-free diet.

* Corresponding author: Dr F. Biagi, fax +39 0382 502618, email f.biagi@smatteo.pv.it 


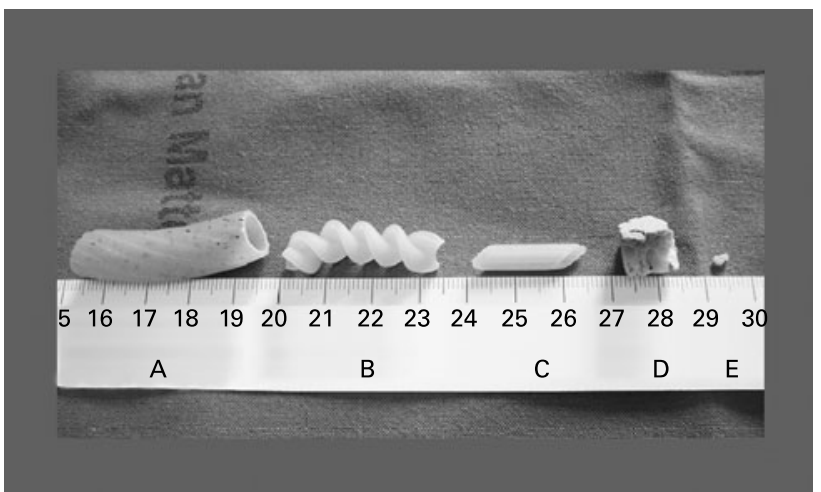

Fig. 1. Different types of commonly consumed gluten-containing food. Samples A, B and C are three different type of pasta, D is a small piece of bread and $E$ is a crumb from $D$. Since gluten is $75 \%$ of the wheat protein ${ }^{(39)}$, according to both the weight and quantity of proteins provided by the producers on the label, sample A contains $209 \mathrm{mg}$ gluten, B $86 \mathrm{mg}$, C $32 \mathrm{mg}$, $D 28 \mathrm{mg}$ and $E 0.5 \mathrm{mg}$. The scale shown is in $\mathrm{cm}$.

Nevertheless, this directive does represent an additional and undeniable safeguard for the health of coeliac patients.

On the basis of this evidence, we believe that the involuntary ingestion of gluten by a patient who is careful about the GFD and his/her health is a limited occurrence and cannot cause significant risks. The aim of the present study was, therefore, to ascertain whether a questionnaire based not on the assessment of how much gluten the patient eats but on the strategies he/she implements to avoid any involuntary ingestion is effective in controlling compliance with the GFD.

\section{Experimental methods}

\section{The questionnaire}

We wanted to devise a simple questionnaire, based on just a few questions, that could be administered in just a few minutes by non-expert personnel. This questionnaire gives a numerical result, in order to monitor the strictness of GFD compliance over time and to be able to compare it between different groups of patients, regardless of nationality. Although the questionnaire was drawn up in the form of an algorithm on the basis of our clinical experience (Fig. 2), the importance of the points we investigated has also been stressed by other authors ${ }^{(23)}$. The questionnaire provides a final score in five levels $(0-\mathrm{IV})$, which from a clinical point of view can be grouped into three levels. Patients with scores of 0 or I are in fact those who do not follow a strict GFD. Patients with scores of II, on the other hand, follow a GFD but with important errors that require correction. Patients with scores of III and IV follow a strict GFD.

\section{Patients}

This was a longitudinal retrospective study in which patient selection was very thorough (thus avoiding a selection bias) and blind (thus avoiding an information bias) ${ }^{(24)}$. The questionnaire was therefore proposed by means of a telephone interview to all the coeliac patients attending our centre who answered the following criteria: (1) diagnosis of coeliac disease based on evidence of villous atrophy at duodenal biopsy and positive anti-endomysial antibodies (EMA) or anti-transglutaminase antibodies; (2) duodenal biopsy and search for EMA at our centre while on a GFD. The purpose of the phone call was explained to the patients and they gave their oral consent to participate in the survey. The present study is part of a larger research programme on coeliac disease carried out by our Institute and approved by the local review board.

Since coeliac patients with minimal intestinal lesions could not be taken into account, 228 patients were found to be affected by coeliac disease on the basis of villous atrophy and positive EMA or anti-transglutaminase antibodies. Forty

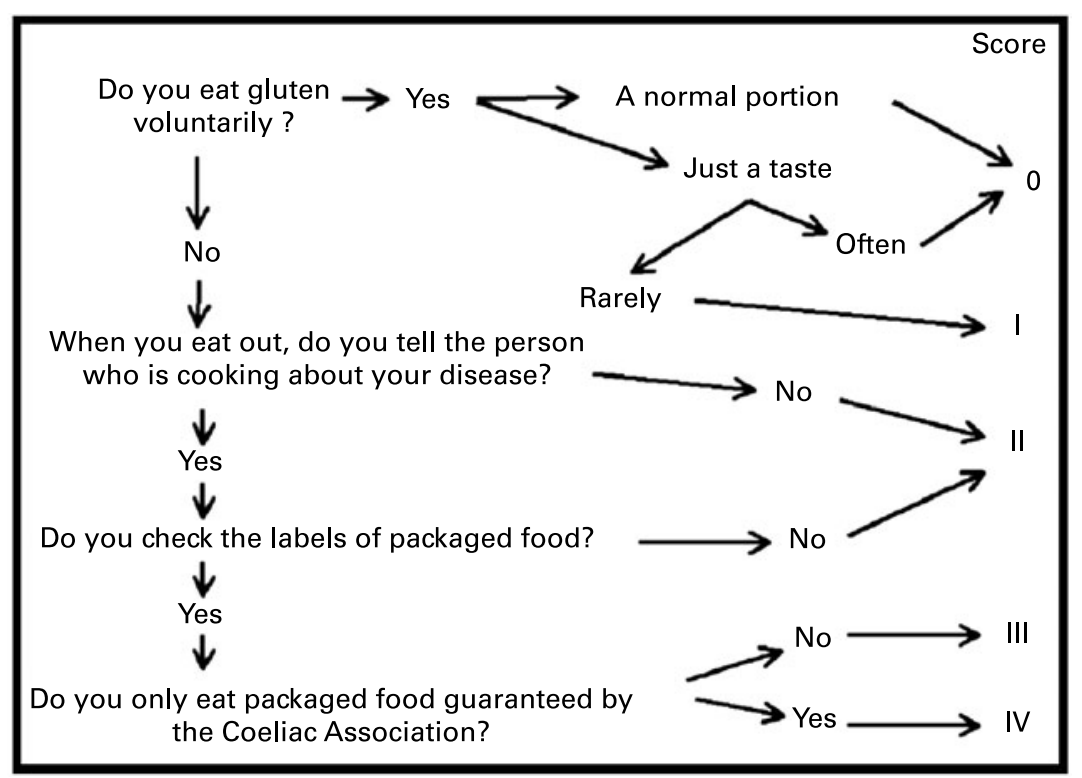

Fig. 2. The questionnaire administered to the coeliac patients. 'Rarely' means that the patient remembers when and how many times he/she has eaten gluten voluntarily. 'Often' means that the patient cannot remember the exact number of times. 
out of the 228 patients had refused to undergo duodenal biopsy and EMA testing while on a GFD. Twenty out of 188 were lost to follow-up. The study was therefore carried out on 168 patients (126 females, aged 42.4 (SD 12.9) years), who had already repeated both duodenal biopsy and EMA testing after a median of 18 months on a GFD (25th-75th percentile 14-40, range 5-327 months). At the time of the interview, they had been on a GFD for a median of 82 months (25th75th percentile 50-108, range 15-389 months).

\section{Validation of the questionnaire}

The questionnaire was validated by comparing the final score across the categories of a series of parameters. First, the score was assessed against the patient's subjective evaluation. In particular, the first question the patient was asked was whether he/she believed they followed the GFD very well, well, badly or very badly. We then assessed the score against the persistence of positive EMA while on a GFD. IgA EMA were detected on monkey oesophagus sections using an indirect immunofluorescence kit (The Binding Site, Birmingham, UK) as previously described ${ }^{(25)}$. We should point out that we normally look for EMA and not for tissue transglutaminase antibodies. A few years ago we showed that in our experience the sensitivity of both EMA and tissue transglutaminase antibodies is higher than $95 \%(25,26)$. We thus prefer to test for EMA, which guarantees $100 \%$ specificity $^{(27)}$. The patients were so classified as either EMA positive or not.

The score was also assessed against the persistence of villous atrophy at duodenal biopsy while on a GFD. Since we were interested in the improvement of the duodenal biopsy, distinguishing the biopsies on the basis of either Marsh or any other morphological classification of coeliac intestinal lesions would have been pointless. The patients were thus classified on the basis of a persistent atrophy of the duodenal mucosa.

Since it has been demonstrated that the strictness of a GFD is one of the factors that establishes the risk of complications and thus the prognosis of coeliac disease ${ }^{(2)}$, we wanted to find out whether the score correlated with the presence of complications and also if it made it possible to predict survival. We therefore included three groups of patients. The first group of nine patients (six females and three males, age 55.6 (SD 11.9) years) had complicated coeliac disease and were still alive: two patients with small-bowel lymphoma, five with ulcerative jejunoileitis, and two with refractory coeliac disease. The second group of nine patients (six females and three males, age 52.9 (SD 11.2) years) had died because of complicated coeliac disease; their scores (and the time of death since the score assessment) were obtained on the basis of their medical records or by interviewing their relatives: four patients with small-bowel lymphoma, one with ulcerative jejunoileitis, and four with refractory coeliac disease. The third group of patients had non-complicated coeliac disease and had responded well to a GFD. In order to prevent selection bias through age at diagnosis ${ }^{(2)}$, this last group was obtained by randomly selecting forty patients diagnosed as adults (age at diagnosis $\geq 30$ years) and who had responded well at histology to a GFD. Since one of these forty patients was among the twenty lost to follow-up, this group actually consisted of thirty-nine patients (twenty-seven females and twelve males, age $48 \cdot 3$ (SD 10.9) years).

Finally, to evaluate the reproducibility of our questionnaire over time, we selected thirty patients with different cultural levels and repeated the questionnaire after an interval of 1 month.

\section{Statistical analysis}

Data were described as medians and interquartile ranges or mean values and standard deviations if continuous and as counts and percentages if categorical. The association of the score with patient characteristics was assessed by means of the Fisher exact test and the test for trend. The role of score on survival was evaluated with the $\log$ rank test and a test for trend in survival. Hazard ratios and $95 \%$ CI were computed by means of Cox regression. Death rates and $95 \% \mathrm{CI}$ per 100 person years were computed.

Stata 10 (StataCorp, College Station, TX, USA) was used for computation. All tests were two-sided. A $P$ value $<0.05$ was considered statistically significant.

\section{Results}

The questionnaire was administered to all the patients by the same non-expert member of the staff (A. A.) in less than 1 min by telephone.

A score of IV was obtained in eighty-three of the 168 patients; a score of III was obtained in fifty-one; II in fifteen, $I$ in six and 0 in thirteen.

As regards the subjective evaluation of the GFD by the patient, by comparing this with the score we observed that $12.8 \%$ of the patients who claimed that they followed the GFD 'very well' or 'well' actually had a score of II, which we consider unsatisfactory.

Table 1. Association of gluten-free score with patient characteristics

\begin{tabular}{|c|c|c|c|c|c|c|}
\hline \multirow[b]{2}{*}{ Characteristic } & \multicolumn{4}{|c|}{ Score } & \multirow[b]{2}{*}{ Fisher exact test: $P$} & \multirow[b]{2}{*}{ Test for trend: $P$} \\
\hline & All & 0 or I & II & III or IV & & \\
\hline EMA-positive while on a GFD (\%) & 30 & 67 & 36 & 24 & 0.001 & $<0.001$ \\
\hline$n$ & $48 / 162$ & $12 / 18$ & $5 / 14$ & $31 / 130$ & & \\
\hline Villous atrophy while on a GFD (\%) & 9 & 45 & 6 & 4 & $<0.001$ & $<0.001$ \\
\hline$n$ & $15 / 168$ & $9 / 20$ & $1 / 16$ & $5 / 132$ & & \\
\hline Complications (\%) & 32 & 100 & 29 & 24 & 0.003 & 0.012 \\
\hline$n$ & $18 / 57$ & $5 / 5$ & $2 / 7$ & $11 / 45$ & & \\
\hline
\end{tabular}

EMA, endomysial antibodies; GFD, gluten-free diet. 
The association of score with patient characteristics is reported in Table 1. Persistence of EMA positivity, of villous atrophy and a presence of complications correlated with our score (Fisher exact tests all $P<0 \cdot 01$ ); moreover the lower the scores, the higher the rate of adverse findings (tests for trend all $P<0 \cdot 05)$. EMA were retested in our laboratory in 162 out of 168 coeliac disease patients. Forty-eight patients still had positive EMA at the time of the duodenal biopsy while on a GFD. The lowest scores were more frequently found in patients with the persistence of EMA positivity. Fifteen patients out of 168 presented persistence of villous atrophy at the time of the duodenal biopsy while on a GFD. The lowest scores were more frequently found in patients with the persistence of atrophy. Although our aim was to validate the score, we should point out that despite strict GFD, villous atrophy was still present in five patients. Two patients had a score of III, and the others a score of IV. In three of them (score III, III, IV) further follow-up biopsies showed that the mucosa had healed. We therefore think that they were 'slow responders $^{\prime(28)}$. The last two patients (score IV, IV) refused to repeat a duodenal biopsy because of very good clinical conditions. Finally, eighteen patients out of fifty-seven in the survival cohort had complications; the lowest scores were observed more frequently in these patients.

Table 2 reports the results of the survival analysis. The score was also associated with survival, with higher death rates in patients with lower scores (log rank test $P=0.005$, test for trend $P=0.008$ ), although when stratifying the analysis for the presence of complications, it was no longer possible to elicit any significant prognostic effect of the score (log rank test $P=0 \cdot 19$, test for trend $P=0 \cdot 15$ ).

Given that complications were highly predictive of death (log rank test $P<0 \cdot 001$ ), in an exploratory subgroup analysis we tested whether, in the eighteen patients with complications, the score could be of use in further stratifying these highly compromised subjects. In fact, a score of 1 or lower was associated with a 3-fold increase in risk of dying (hazard ratio $3.3 ; 95 \%$ CI $0.72,16.7$ ), although, given the low power available, statistical significance was not reached (log rank test, $P=0 \cdot 08$ ).

Patients from different social groups and with different cultural levels who answered the questionnaire all stated that they had no difficulty in understanding the questions. In the thirty patients who repeated the test after 1 month, we found an almost perfect agreement, with a $\kappa$ statistic of 0.91 .

Table 2. Association of gluten-free score with mortality

\begin{tabular}{lccc}
\hline & \multicolumn{3}{c}{ Score } \\
\cline { 2 - 4 } Survival analysis & 0 or I & II & III or IV \\
\hline Number of deaths & 5 & 0 & 4 \\
$\quad n$ & 100 & 0 & 9 \\
$\%$ & 187 & 0 & 15 \\
Rate per 100 person years & 78,450 & & 5,39 \\
$\quad 95 \%$ Cl & & 0.005 & \\
Log rank test: $P$ & & 0.008 & \\
Test for trend in survival: $P$ & & 0.19 & \\
Log rank test (stratified by & & 0.15 & \\
$\quad$ complication): $P$ & & & \\
Test for trend in survival (stratified by & & & \\
$\quad$ complication): $P$ & &
\end{tabular}

\section{Discussion}

Coeliac disease is a very common condition that requires an expensive and lifelong dietary therapy. So, to avoid both recurrence of intestinal lesions and wasting money, it is very important to verify whether the patients are actually on a strict diet. However, although numerous methods have been proposed to assess compliance with a GFD (for a review, see Pietzak ${ }^{(29)}$ ), we still do not know which of these is the best method. Although the impossibility of knowing exactly what and, above all, how much an individual eats has already been pointed out ${ }^{(30)}$, the current 'gold standard' for assessing compliance with a GFD is data collection by the patient which is then discussed during a diet interview. At the end, a doctor or an expert dietitian expresses a subjective evaluation on the patient's compliance with the GFD ${ }^{(29)}$. This data collection can be in the form of a food record or a food questionnaire ${ }^{(30)}$. The food record consists of the patient's record, over a set period of time, of all the food and drink consumed during the day. The food questionnaire, on the other hand, assesses how many times a certain food or drink is consumed in the set period of time, regardless of the quantity. However, in analysing thirteen articles in which these diet assessment systems are applied in patients with coeliac disease, we observed that a standardisation of the method is completely lacking ${ }^{(4-8,10,30-36)}$. The data were in fact collected in at least eight different ways and only two of these articles report the questions that the patients were asked ${ }^{(30,31)}$. The food record and food questionnaire have additional limitations. It is not easy for a patient to keep a food record and he/she can only do so for a few days, which is of little use in the context of a disease which requires a lifelong diet; the patient tends to be more careful with his/her diet on the days the diary is kept; the diary is geared towards evaluating the frequency of gluten intake without providing a quantitative assessment, unless the food is weighed, an impractical task which in any case would not reflect the usual diet. Finally, both the food questionnaire and the food record then need to be analysed by expert personnel with the same 'culinary background' as the patient. Such personnel can certainly provide a reliable assessment of the patient's compliance with the GFD, but this assessment will inevitably be subjective and not comparable with assessments made by others.

With the present study we succeeded in creating a system that evaluates the compliance with a GFD of patients with coeliac disease by means of an extremely rapid and simple numerical score that can also be obtained by personnel without specific experience in coeliac disease. The numerical result thus makes it possible to monitor the patient's compliance with the GFD over time and to make comparisons between different groups of patients. This possibility of comparing different groups of patients is made even easier by the fact that our score is not based on an assessment of what the patient eats, which would make it very difficult to compare patients from different countries or with different cultural backgrounds, but on the strategies that the patient uses to avoid eating gluten, regardless of the actual food eaten.

In order to validate the questionnaire, it was compared with other methods. We first assessed it against the histological and serological response to the GFD. Histological response is certainly the most valid method for evaluating compliance with a 
$\mathrm{GFD}^{(29)}$. Although serology is not sensitive enough to detect the occasional intake of gluten ${ }^{(37)}$, it is certainly one of the most commonly used methods ${ }^{(29)}$. In addition, since it has already been demonstrated that poor compliance with a GFD is a negative prognostic factor ${ }^{(2)}$, we related the score to the presence of complications and with patient survival. The score was associated with survival, but this association disappeared when accounting for complications. However, we performed a subgroup exploratory analysis in patients with complications; in these patients, we showed that the score might have an additional prognostic role, increasing the risk of dying by 3 . The low power prevented us from eliciting statistical significance.

In the present study, we demonstrated that our score correlates to a statistically significant extent with all these parameters, and that higher prevalences of adverse findings are associated with lower scores.

In validating the score we were obviously unable to make a comparison with a classic dietary interview, as this would have included an obvious bias in the study. When we planned the study, we wondered about whether to also compare the score with the clinical response to a GFD. Of the patients who took part in the study, $50 \%$ presented a minor or silent clinical form of coeliac disease ${ }^{(1)}$. Moreover, we recently described a case report in which eating a 'low-gluten-containing diet' was followed by a complete clinical and serological response but a lack of histological response ${ }^{(38)}$. We therefore believe that this clinical parameter was very difficult to measure and we therefore preferred not to take it into consideration.

The present study obviously also has limitations. First of all, the size of the study population is relatively small, above all as regards the study of survival. Although we performed the study blind and with great care, it is a longitudinal retrospective study, which means that the patients answered the questionnaire some time after the serological and histological tests had been carried out (median 53; 25th-75th percentile 29-72 months). Moreover, the patients were not blinded as they knew the results of the EMA and their biopsy before the survey. This may have introduced an information bias, as the patients who were found to be positive either by assay or histology are likely to have recalled about the compliance to the diet and are more likely to have recognised errors. The score itself also has certain essential limitations, such as the necessity that the patient has been appropriately instructed by an expert dietitian as to what a GFD really means and the fact, not always the case, that the patient is telling the truth. A further limitation of the study was the need, in the deceased patients, to collect information for calculating the score from notes and relatives.

Despite these limits, we believe that these results are nevertheless very satisfactory and promising, and that they provide the basis for a longitudinal prospective validation.

\section{Acknowledgements}

We are indebted to the Coeliac Society of Western Australia, the Associazione Italiana Celiachia, Coeliac UK and the Celiac Sprue Association for their help with food labelling regulations.
We are grateful to Susan West for reading and correcting the manuscript.

F. B. designed the study, interpretated the results and wrote the manuscript. A. A. administered the questionnaire to the patients. P. I. B. tested EMA. A. M. acquired the clinical and histological data. C. K. performed the statistical analysis. G. R. C. participated in the design of the study and in the critical revision of the manuscript.

We declare that we have no conflicts of interest and no funding for the present study.

\section{References}

1. Biagi F \& Corazza GR (2002) Clinical features of coeliac disease. Dig Liv Dis 34, 225-228.

2. Corrao G, Corazza GR, Bagnardi V, et al. (2001) Mortality in patients with coeliac disease and their relatives: a cohort study. Lancet 358, 356-361.

3. Case S (2005) The gluten-free diet: how to provide effective education and resources. Gastroenterology 128, S128-S134.

4. Mayer M, Greco L, Troncone R, et al. (1991) Compliance of adolescents with coeliac disease with a gluten free diet. Gut 32, 881-885.

5. Ljungman G \& Myrdal U (1993) Compliance in teenagers with coeliac disease - a Swedish follow-up study. Acta Paediatr 82, $235-238$.

6. Greco L, Mayer M, Ciccarelli G, et al. (1997) Compliance to a gluten-free diet in adolescents, or "what do 300 coeliac adolescents eat every day?'. Ital J Gastroenterol Hepatol 29, 305-310.

7. Rashid M, Cranney A, Zarkadas M, et al. (2005) Celiac disease: evaluation of the diagnosis and dietary compliance in Canadian children. Pediatrics 116, e754-e759.

8. Karajeh MA, Hurlstone DP, Patel TM, et al. (2005) Chefs' knowledge of coeliac disease (compared to the public): a questionnaire survey from the United Kingdom. Clin Nutr 24, 206-210.

9. Ciacci C \& Mazzacca G (1998) Unintentional gluten ingestion in celiac patients. Gastroenterology 115, 243.

10. Collin P, Thorell L, Kaukinen K, et al. (2004) The safe threshold for gluten contamination in gluten-free products. Can trace amounts be accepted in the treatment of coeliac disease? Aliment Pharmacol Ther 19, 1277-1283.

11. Jansson UH, Gudjónsdóttir AH, Ryd W, et al. (2001) Two different doses of gluten show a dose-dependent response of enteropathy but not of serological markers during gluten challenge in children with coeliac disease. Acta Paediatr 90, $255-259$.

12. Mothes T \& Stern M (2003) How gluten-free is gluten-free, and what does this mean to coeliac patients? Eur J Gastroenterol Hepatol 15, 461-463.

13. Hischenhuber C, Crevel R, Jarry B, et al. (2006) Review Article: safe amounts of gluten for patients with wheat allergy or coeliac disease. Aliment Pharmacol Ther 23, 559-575.

14. Catassi C, Fabiani E, Iacono G, et al. (2007) A prospective, double-blind, placebo-controlled trial to establish a safe gluten threshold for patients with celiac disease. Am J Clin Nutr $\mathbf{8 5}$, $160-166$.

15. Catassi C, Rossini M, Rätsch IM, et al. (1993) Dose dependent effects of protracted ingestion of small amounts of gliadin in coeliac disease children: a clinical and jejunal morphometric study. Gut 34, 1515-1519.

16. Gibert A, Espadaler M, Angel Canela M, et al. (2006) Consumption of gluten-free products: should the threshold value for trace amounts of gluten be at 20,100 or 200 p.p.m.? Eur J Gastroenterol Hepatol 18, 1187-1195. 
17. Celiac Sprue Association (2009) FDA Definition of Gluten-free Labeling. http://www.csaceliacs.org/FDADefinitionofGlu tenfreeLabeling.php\#Allergen

18. Coeliac UK (2009) Coeliac UK - the charity for people with coeliac disease and dermatitis herpetiformis. http://www. coeliac.co.uk

19. The Coeliac Society of Western Australia (2009) The Coeliac Society of Western Australia. http://www.wa.coeliacsociety. com.au

20. Associazione Italiana Celiachia (2009) Associazione Italiana Celiachia. http://www.celiachia.it

21. European Commission (2003) Directive 2003/89 EC of the European Parliament and of the Council of 10 November 2003 amending Directive 2000/13/EC as regards indication of the ingredients present in foodstuffs. Official Journal of the European Union, L308, pp. 15-18. http://eur-lex.europa.eu/LexUriServ/ LexUri Serv.do?uri=CELEX:32003L0089:EN:HTML

22. US Food and Drug Administration (2004) Food Allergen Labeling and Consumer Protection Act of 2004 (Title II of Public Law 108-282). http://www.cfsan.fda.gov/ dms/alrgact.html

23. See J \& Murray JA (2006) Gluten-free diet: the medical and nutrition management of celiac disease. Nutr Clin Pract 21, 1-15.

24. Rothman KJ (1996) Modern Epidemiology. Boston and Toronto: Little, Brown and Company.

25. Biagi F, Pezzimenti D, Campanella J, et al. (2001) Endomysial and tissue transglutaminase antibodies in coeliac sera: a comparison not influenced by previous serological testing. Scand $J$ Gastroenterol 9, 955-958.

26. Biagi F, Ellis HJ, Yiannakou JY, et al. (1999) Tissue transglutaminase antibodies in celiac disease. Am J Gastroenterol $\mathbf{9 4}$ 2187-2192.

27. Biagi F \& Corazza GR (2001) Tissue transglutaminase antibodies: is sensitivity more important than specificity? Dig Liver Dis 33, 401-402.

28. Grefte JM, Bouman JG, Grond J, et al. (1988) Slow and incomplete histological and functional recovery in adult gluten sensitive enteropathy. J Clin Pathol 41, 886-891.
29. Pietzak MM (2005) Follow-up of patients with celiac disease: achieving compliance with treatment. Gastroenterology 128, S135-S141.

30. Hopman EG, Kiefte-de Jong JC, le Cessie S, et al. (2007) Food questionnaire for assessment of infant gluten consumption. Clin Nutr 26, 264-271.

31. Baker PG, Barry RE \& Read AE (1975) Detection of continuing gluten ingestion in treated coeliac patients. $\mathrm{Br}$ Med $\mathrm{J} \mathbf{1}$, 486-488.

32. Fabiani E, Catassi C, Villari A, et al. (1996) Dietary compliance in screening-detected coeliac disease adolescents. Acta Paediatr Suppl 412, 65-67.

33. Van Overbeek FM, Uil-Dieterman IG, Mol IW, et al. (1997) The daily gluten intake in relatives of patients with coeliac disease compared with that of the general Dutch population. Eur J Gastroenterol Hepatol 9, 1097-1099.

34. Bardella MT, Fredella C, Prampolini L, et al. (2000) Body composition and dietary intakes in adult celiac disease patients consuming a strict gluten-free diet. Am J Clin Nutr 72, 937-939.

35. Mustalahti K, Lohiniemi S, Collin P, et al. (2002) Gluten-free diet and quality of life in patients with screen-detected celiac disease. Eff Clin Pract 5, 105-113.

36. Faulkner-Hogg KB, Selby WS \& Loblay RH (1999) Dietary analysis in symptomatic patients with coeliac disease on a gluten-free diet: the role of trace amounts of gluten and non-gluten food intolerances. Scand $J$ Gastroenterol 34, 784-789.

37. Troncone R, Mayer M, Spagnuolo F, et al. (1995) Endomysial antibodies as unreliable marker for slight dietary transgressions in adolescents with coeliac disease. J Pediatr Gastroenterol Nutr 21, 69-72.

38. Biagi F, Campanella J, Martucci S, et al. (2004) A milligram of gluten a day keeps the mucosal recovery away. Nutr Rev 62, 360-363.

39. Shewry PR, Halford NG, Belton PS, et al. (2002) The structure and properties of gluten: an elastic protein from wheat grain. Philos Trans R Soc Lond B Biol Sci 357, 133-142. 